\title{
A Review of Various Routing Protocols in VANET
}

\author{
Shivani Rana \\ M.Tech Scholar \\ Graphic Era Hill University \\ Dehradun, Uttrakhand - India
}

\author{
Swati Rana \\ Lecture \\ Sunrise Institute of \\ Management \& Sciences \\ Dehradun, Uttrakhand - India
}

\author{
Kamlesh C. Purohit \\ Assistant professor \\ Graphic Era University \\ Dehradun, Uttrakhand - India
}

\begin{abstract}
Vehicular Ad-hoc Network (VANET) is a self-organized network that connects vehicle and RSUs. The RSUs can intern be connect to a background network so that many other network applications and services including internet access can be provide to the vehicle for obtaining different services by the user or driver. The considerable attention goes in this field due to the high demands of new innovations in the vehicular industry. The primary purpose of VANET is to improve public safety and save lives as well as to improve vehicular traffic flow. VANET has very dynamic topology large network size and constrained mobility, these characteristic led to the need for efficient routing and resource saving protocol. It is a new research area which tends lots of emphasis towards services provided through the network. In this paper, we give the review of various routing protocols by using some parameters: digital map, scenario, position verification, clustering, routing mechanism, forwarding strategy and control overhead.
\end{abstract}

\section{General Terms}

Mobile Ad-Hoc Network (MANET), Vehicular Ad-Hoc Network (VANET), Protocols.

\section{Keywords}

Vehicular Ad-hoc Network (VANET), RSUs, Routing protocols.

\section{INTRODUCTION}

There are many types of infrastructure-less network i.e. adhoc network, it is a collection of wireless mobile nodes without any fixed infrastructure. Mobile ad-hoc network (MANET) is a sub class of ad-hoc network. Vehicular ad-hoc network (VANET) is a distinctive class of mobile ad-hoc network (MANET) which accommodates different approach for Intelligent Transport System (ITS)[1]. But presently VANET have become more critical research and development area which allows communication between vehicle to vehicle and vehicle to Road Side Unit (RSUs) through a wireless sensing device which is installed in each vehicle in the network.

VANET is one of the most challenging areas due to very high and unpredictable dynamic topology and frequent disconnections. It provides safety and security in vehicular system. VANET supports two types of communication: Vehicle to vehicle and vehicle to infrastructure communication. In vehicular communication, information generation and distribution occur with the vehicle to vehicle and vehicle to infrastructure $[2,3]$

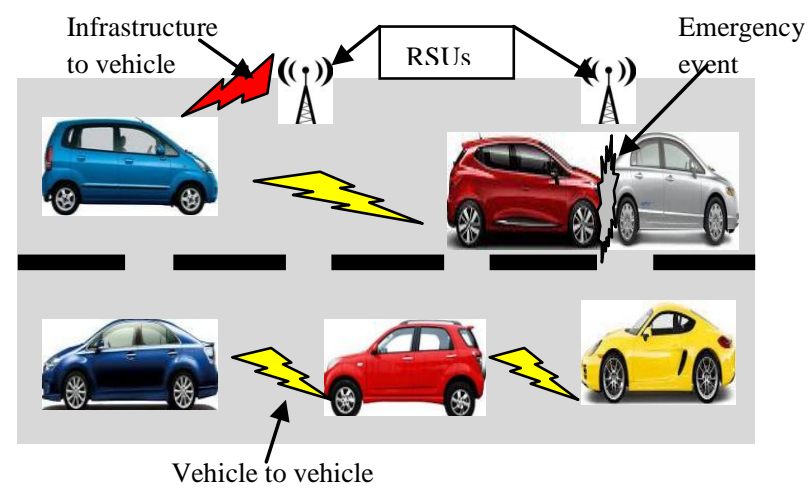

Figure 1: VANET Architecture

VANET has following different features:

a. The ability of moving vehicles is highly predictable because vehicles are moving with only two directions on the same road.

b. Vehicles provide lots of electric power to the wireless sensing devices which are already present in the vehicles.

c. In VANET, broadcast communication is used to deliver information from sender to receiver instead of unicast communication.

Plenty of routing protocols have been constructed for MANET. But MANET routing protocol does not suitable for VANET because of high speed of vehicles and active information transfer. Routing in VANET is a challenging task due to unique characteristics like high mobility, and frequent network disconnection. In order to provide stable route and obtain good performance in VANET proper routing protocol must be designed. VANET has distinct architecture that allow the communication between vehicles and vehicle to RSUs leading to the following three possibilities [3, 4].

Vehicle to Vehicle ad-hoc network architecture: This is also known as pure ad-hoc network which allows direct vehicular communication without need of any fixed infrastructure support.

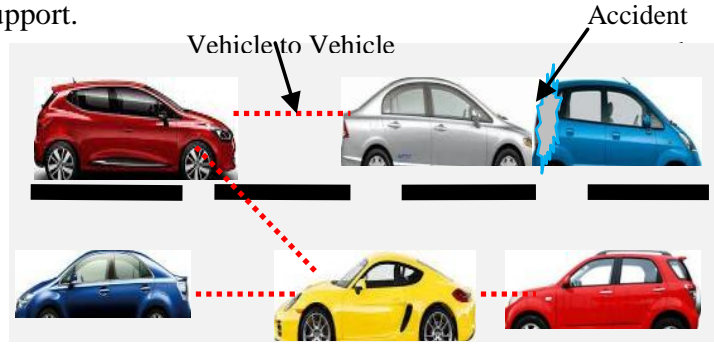

Figure 2: Vehicle to Vehicle Architecture 
Vehicle to Infrastructure network architecture: This type of network use a cellular gate way and wireless local area network access point to allow a vehicle to communicate with the roadside units mainly for information and data gathering application.

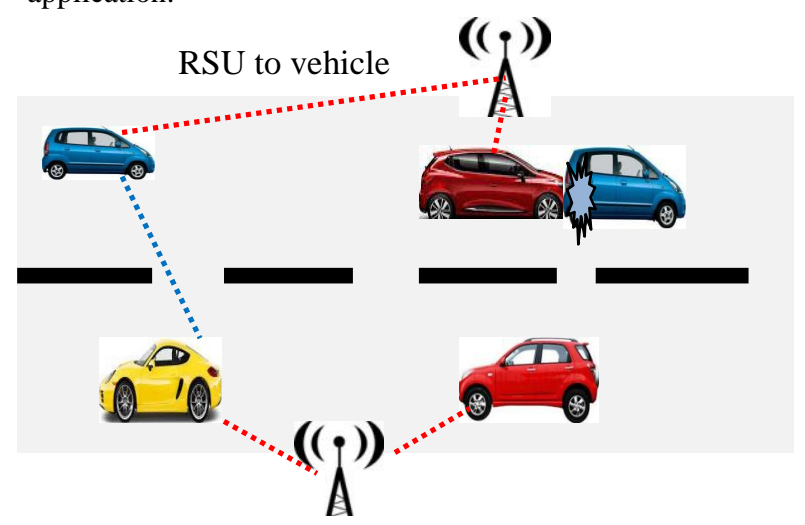

Figure 3: Vehicle to Infrastructure Architecture

Hybrid architecture: This combines both Vehicle to Vehicle ad-hoc networks (VtoV) and Vehicle to Infrastructure network architecture (VtoI).

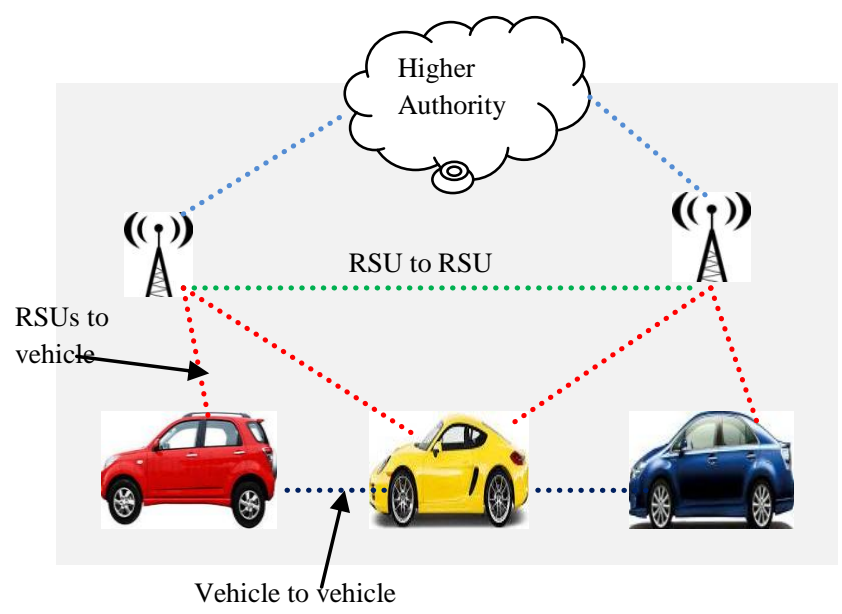

Figure 4: Hybrid Architecture

\section{CHARACTERISTIC AND CHALLENGES OF VANET}

\subsection{Characteristics of VANET [2, 5]:}

Dynamic topology-One of the most important characteristics of VANET is dynamic topology. In this nodes or vehicles move with high speed in respect to each other.

No power constraints and adequate storage -In VANET, we are using vehicles as nodes instead of other devices so vehicles have sufficient amount of energy and power including both processing and storage; so the battery power and storage is not an issue in VANET.

Frequent network disconnection-In VANET, vehicles move very frequently on the roads, in the network due to this the link connectivity in VANET also change frequently. The chances of network disconnection are high when the density of vehicle is low.

Mobility modeling and prediction-Due to high dynamic topology, mobility modeling and prediction play very important role for designing the data dissemination in VANET.
Different communication environments-VANET has two types of environments i.e. highway environment \& city environment. In highway traffic environment, the communication is comparatively simpler and straight forward. But in city traffic environment It become quite complex as compare to highway traffic environment.

\subsection{Challenges of VANET [6, 7]:}

Hidden terminal problem- This problem may occurs when two or more objects sends packets, these packets are not within the direct transmission range of each other. Collide at the common receiver node.

Error prone shared ratio- In VANET, during propagation the radio wave go through several impairments such as attenuation, multipath propagation and interference.

Insecure medium- In VANET, due to broadcasting nature communication is not secure. It is hard for these networks to support different applications with appropriate QoS requirements.

Lack of central coordination- In VANET, there is no central controllers to coordinate the activity of nodes.

Dynamic varying network topology- In VANET, the network topology changes dynamically with high speed. Due to this QoS session may suffer frequently path break.

\section{CATOGORIES OF ROUTING PROTOCOL}

MANET routing protocols are not suitable for VANET because MANET routing protocols has difficulties for finding suitable routing path in VANET. In VANET routing protocol can be divided into five groups. These are Topology based routing protocol, Position based routing protocol, Geo-cast based routing protocol, Cluster based routing protocol, and Broadcast based routing protocol $[8,9]$

Topology based routing protocol [9] use links information which stored in the routing table that exists in the network to forward data packet from sender to receiver. They can be commonly divided into proactive (table driven) routing protocol, reactive (on demand) routing protocol, hybrid routing protocol.

Proactive routing protocols $[10,11]$ usually based on the shortest path algorithm to determine which route will be chosen. These protocol use routing table to store routing information and routing table also keep information of all connected nodes and when network topology face any changes every node updates its routing table.

Reactive routing protocols $[10,11]$ are on demand routing protocol because it starts route discovery only when a node wants communicate with another node. These protocols reduce the burden in the network.

Hybrid routing protocols $[10,11]$ are combined the properties of both proactive and reactive routing protocol. The aim of hybrid routing protocol is to minimize the control overhead of proactive routing protocol and reduce the delay of route discovery process in reactive routing protocol. In hybrid routing protocol, the network is divided into many zones and it provide more reliability for route discovery and maintenance process.

Cluster Head Gateway Switch Routing (CGSR) [10] is a proactive clustered multi hop wireless. This protocol is dissimilar outside other protocol in the type of dispatching and network configuration. CGSR exploit better bandwidth 
and compress the bigness of distance vector table because it is performed only over cluster head. But the shortcoming of CGSR is that it consume more time for selecting a cluster head and gateway.

Destination Sequenced Distance Vector Routing (DSDV) [10] is use to resolving the worriment which is identified with the distance vector routing of wired network. This protocol solves the worriment by using destination sequence number. It uses shortest path algorithm and provides one route to every node from sender to receiver which is loop free. In DSDV each routing table contains information about all nodes which accessible in the network and hops that are needs to reach that node. Each node broadcast its routing table to its neighbor for maintaining the route reliability.

Optimized Link State Routing Protocol (OLSR) [11] apparatus the link state approach. OLSR maintains a routing table which accommodates information about all possible routes to network. When the network topology changed, each node must send it updated information to all other some selective nodes and these selective nodes retransmit these updated information to its other selective nodes. But some nodes which are not in the selected list can only read and process the packet. Some researches analyze that OLSR routing protocol has easily done procedure which acquiesce it to build in different operating system. It performs well in the dynamic topology and it also suitable for data transmission applications which required low latency.

Fisheye State Routing Protocol (FSR) [11] is an enhancement of global routing protocol and also called reactive routing protocol. In this each node knows about is neighbor node and calculate the routing table. FSR reduced the consumed bandwidth by exchanging the routing information only with the neighbors. The disadvantage of FSR is that, if the network size increases then the size of routing table also increases and if the changes of topology increased, the route from sender to receiver can be inaccurate.

Ad Hoc on Demand Distance Vector Routing Protocol $(A O D V)$ [11] is on demand routing protocol because in AODV, route is generated when a node wants to send data packet with another one. AODV can be used either unicast or multicast communication. In AODV when a node requested for a route then route discovery process is active. Once route is created the route maintenance procedure maintains the route and route discovery procedure comes to end. In AODV each node maintains the routing table which holds the value of next hope node, sequence number and a hop count. The problem with this protocol is that, a node has to wait for some time to find the route from source to destination so this protocol cannot be suitable for time critical and safety related application.

Dynamic Source Routing Protocol (DSR) [12] is a multi-hop reactive routing protocol. DSR scaled down the network overhead by contracting periodic messages. This protocol applies source routing and maintains active route. DSR protocol subsists of two operations: route discovery and route maintenance that makes DSR protocol to self-configuration and self-organization. It is network type adjustability in which every packet hold complete successful route to the destination to in its cache. But if any failed route occurs, this protocol will replace it by another successful route. But in DSR protocol, the Route Maintenance mechanism does not reconstruct the broken link.
Dynamic MANET on Demand Routing Protocol (DYMO) [13] is an efficient reactive routing protocol that specially designed for use in multi-hop wireless network. This is simple routing protocol and easy to implement. DYMO also consists of two basic operations: Route Discovery and Route Maintenance.

Temporally Ordered Routing Algorithm (TORA) [14] is distributed routing protocol and uses multi-hop routes. It is part of the family of link reversal routing. In this each node build the direct acyclic graph (DAG). Direct acyclic graph is constructed by broadcasting query packets by each node. When another node receiving that query packet and has a route to the destination it will send back the reply packet otherwise it drop the packet. The advantage to TORA is that it allows a route to all presented node in the network and disadvantage is that the route maintenance is difficult in VANET.

Zone Routing Protocol (ZRP) [14] is a first hybrid routing protocol. In ZRP, a network is divided into number of zones and each zone is a collection number of nodes. The size of each zone is decided by the radius of length $\mathbb{a}$. Where $(\mathbb{\alpha})$ can be expressed as the number of hops to the perimeter of the zone.

$\alpha=\frac{\text { number of hops }}{\text { perimeter of the aone }}$

In ZRP, reactive routing protocol is used outside the zone and proactive routing protocol is used inside the zone. ZRP adopt the broadcasting scheme to build multicast trees to flood the query packet and the goal of ZRP is to find loop free roots from source to destination.

Hybrid ADHOC Routing Protocol (HARP) [14] is another type of hybrid protocol in this a network is divided into number of non-overlapping zones. The objective of HARP is to reduced delay by establishing a stable route from source to destination. This chose best route which is based on stability criteria. In HARP, the routing is implementing into two levels: intra zone and inter zone. Intra zone using proactive routing and inter zone using reactive routing. This protocol is suitable for urban network but does not suitable for high mobility ad-hoc network.

Position Based Routing Protocol [9] is type of protocol that requires a position of determining services like GPS. In this each node knows is own and neighbors geographical position by GPS. Position based routing is suitable for high dynamic mobility pattern and does not require any routing table. Position based routing can be divided into two types of protocol: position based greedy $\mathrm{V} 2 \mathrm{~V}$ protocol and delay tolerant protocol.

Greedy Perimeter Coordinator Routing Protocol (GPCR) [15] is topology based routing protocol that adopts coordinators for forwarding the data. Coordinators can be defined as a node which stationed beside the junctions. This protocol uses the approach of sending the data packet to a node on the junction not across the junction. This approach gives guarantee that the packet will be transmitted in a right direction. It is used greedy strategy for packet forwarding. The advantage of this protocol is that it does not depend upon the global information. It does not have unidirectional links and planner sub-graph problem.

Greedy Perimeter Stateless Routing Protocol (GPSR) [14] is position based routing protocol that is suitable for highway. In GPSR, each node systematically broadcast a beacon message 
to all neighbors. Each beacon message holds the id and position of respective node. If any node does not receive any beacon message from its neighbor in a given time, it assume that the neighbor has been abort or outside of limit and GPSR router delete the neighbor from its table. It uses greedy forwarding mechanism for taking information about next node i.e. neighbor in the network. If greedy forwarding strategy is infeasible for any node then it takes perimeter of the region strategy to know about next forwarding hop. GPSR protocol relies upon two approaches: Greedy forwarding approach (the node directly forwards the data packet to its closer destination node) and Recovery approach (this approach is used to forward a packet to the closer destination where packet comes up the local maximum).

Connectivity-Aware Routing Protocol (CAR) [16] [17] is topology based routing protocol that has four parts, these are destination location \& route selection process, data forwarding with the path, path maintenance using the concepts of 'Guards' and error recovery. CAR associate a location service into its route selection process, used new beaconing mechanism and route recovery approach. This uses PGP to identify time location of destination. In CAR, every node broadcast the route discovery request with its data and each forwarding node updates the three fields: number of hops, average number of neighbors and minimum number of neighbors and send back a route reply to source with the information of destination location and velocity vector. Anchor points are built from sender to receiver at the same time. But if link break occurs CAR uses the concept of standing 'Guards'. The purpose of guards is to maintain the routes from sender to receiver and repair them if it breaks. CAR improves the packet delivery ration and shows lesser routing overhead as compare to GPSR.

Geographical Source Routing Protocol (GSR) [18] is geographical source routing protocol that tries to conquer the drawback of the geographical routing by combining the location based routing with topological information. It uses reactive location service (RLS) to get the information of the destination's location and uses digital map to select the path from sender to receiver. It uses dijkastra's algorithm for calculating the smallest path from sender to receiver. In path selection, all the junctions to be gone over from source to destination are selected. Source node added to the list of junction to the packet header or intermediate nodes come to decision about the junction near the destination. The packet is forwarded in greedy manner in between selected junctions.

Anchor-Based Street and Traffic Aware Routing Protocol (A$S T A R$ ) [19] is location based routing protocol and specially designed for city scenario for inter vehicle communication system. It also uses new local recovery approach which is suitable for city scenario and calculates a new anchor path from the local maximum from which the packet is routed. This protocol also defines the maximum number of times a packet can be recovered so as avoid out dated packet being transmitted repeatedly. In A-STAR, routes are based on two types of maps: a statically rated map and a dynamically rated map.

Contention Based Forwarding Routing Protocol (CBF) [20] is a geographical routing protocol. It does not make use of the transmission of beacon messages. In CBF, if any node wants to send the data packet, it will broadcast the data packet to all direct neighbors. These nodes select the next node through a distributed contention process. This contention process requires the biased timers. This protocol saves the bandwidth due to elimination of beacon message and also decreases the probability of packet collision by avoiding faulty neighbors.

Street Topology Based Routing Protocol (STBR) [20] is based on illustrate given street map. STBR hold three valid states: one is master, second is slave and third is forwarder. It computes the node connectivity at the junction nodes. In STBR, master state is responsible for checking, if links to the next junction are up and down and forwarder is an intermediate node between junctions.

Border-Node Based Most Forward within Radius Routing Protocol (B-MFR) [20] is used to minimize the number of nodes between sources to destination by using the concept of border node in the period of sender communication range. BMFR makes use of border node to prevent utilization of internal nodes inside the transmission range for dispatching the packet. It using next hop forwarding method which is not support in highly mobile ad hoc network such as VANET for linear network.

Geo-cast Based Routing Protocol [9] is a multicast routing protocol that is location based. The main aim of this protocol is to forward a data packet from sender node to all other nodes in the geographical region.

Inter-Vehicle Geo-cast Routing Protocol (IVG) [9] broadcast the safety message to the vehicles in the network when any accident occurs on the highway. This protocol adopt a mechanism which is timer based for message forwarding. This protocol also adopt periodic broadcast i.e. used for reduced network fragmentation. In this protocol, a busted vehicle disseminate an alert message to all vehicles in the group and the neighbor which received alert message analyze its applicability based on their location informing to the risk area.

Robust Vehicular Routing Protocol (ROVER) [9] is geographical multicast protocol. In ROVER, control packets are using broadcasting and data packets are using unicasting mechanism. The main goal of this protocol is to broadcast a message to all presented vehicles in the specified zone of relevance (ZOR). ZOR can be designate as a rectangle specified by its corner coordinates. In this, a message can be described by using three terms called triplet (application, message, ZOR). If the vehicle within its $\mathrm{ZOR}$ and received a message then it accepts that message.

Dynamic Time-Stable Geo-cast Routing Protocol (DTSG) [9] is the type of protocol which depend on network density and speed of vehicles foe better performance. This protocol is used to inform the vehicle about accident which associate with the particular region on the highway in a certain period of time. There are two phases defined by the DTSG: Prestable (This phase is used for broadcast the message until it reached the end region) and stable-period (This phase helps to intermediate node which uses to store and forward method for predefined time within given region).

Direction-Based Geo-cast Routing Protocol for Query Dissemination (DG-CASTOR) [9] is based on link availability estimation. It is used to estimate the neighbors, which neighbors have same ability to communicate with sender in given period of time. It is especially designed for commercial purpose in VANETs. The main aim of DG-CASTOR is to build an essential commonality that is based on future location prediction of moving nodes in the network. This commonality is known as Rendez-vous group represents the geo-cast region where the ability of communication of nodes may estimate. 
Distributed Robust Geo-cast Routing Protocol (DRG) [20] defines Zone of relevance (ZOR) and Zone of forwarding (ZOF) for improving the reliability of message forwarding. ZOR the set of all nodes for which the message is relevant and ZOF the collection of nodes which appropriate for forwarding the geo-cast message. DRG protocol works in manner if a vehicle receives geo-cast message then it checks its relevance according to its location and it found that the vehicle belongs to the ZOF then it either forward the message or drop the message and DRG does not require any exchange of periodic beacons messages.

Cluster Based Routing Protocol [21] is the type of cluster based protocol in which the network is separated into number of clusters. Each cluster has number of nodes with same characteristics like same direction \& same velocity. Each cluster also maintains the cluster head and the function of cluster head is to control the communication process with inside the cluster and outside the cluster. If a node wants to communicate with another node inside the cluster it uses direct path, but if a node wants to communicate with other node outside the cluster it used cluster head.

Clustering for Open IVC Network Routing Protocol (COIN) [12] is a cluster based protocol because it uses clustering mechanism for improving network scalability. It divides the network into different cluster. In COIN, the selection of cluster is depending on the three different parameters: movements of nodes, position of nodes, and behavior of nodes. In this protocol, each cluster has a specific time that called time to live and it is used to reduce control overhead. The mobility of nodes should be low due to that nodes can communicate with each other for long time.

Cluster-Based Directional Routing Protocol (CBDRP) [14] is a cluster based routing protocol which divides the network into cluster on the basis of same direction vehicle means (which vehicle have the same direction they could be come in same cluster). Source node send packet to its cluster head and cluster head send packet either to the vehicle which is in the same cluster or forward the packet to cluster head of another cluster and this cluster head forward the packet to the destination. It works same as CBR i.e. selection and maintenance of cluster head is same as CBR but it select the cluster head by velocity and direction of vehicle.

Cluster Based Routing Protocol (CBR) [18] is geographical cluster based protocol that divides the network into number of square grids. It does not require discovering the route from sender to receiver. This protocol is basically used the position of the vehicle. In this, each node forward the data from one node to next node by using geographical information and a vehicle that is selected as a cluster head in a grid, will broadcast LEAD message to all its neighbors. Before leaving the grid the cluster head forward the LEAVE message to all neighbors. The disadvantage of CBR is that it does not consider main two important parameters i.e. Velocity \& direction for VANETs.

Cluster Based Location Routing Protocol (CBLR) [14] is on demand cluster based routing protocol. In CBLR, each cluster head maintains two tables: table one contains the address and position of cluster member and gateways nodes and another table contains the information about neighboring cluster i.e. called cluster neighbor table. When source node and destination node is in same cluster, source node simply forward data to the closest neighbor of the destination. But id the source not and destination is not in the same cluster, source node store the data in its buffer and broadcast location request packet (LREQ) and start timer. When cluster head receive the request message it will check whether it is in its cluster or not, if it is a cluster member then it forward the location reply (LREP) to the sender. CBLR protocol is basically used for high mobility network because it regular updates the position of source and destination before transmitting the data.

Hierarchical Cluster Based Routing Protocol (HCB) [14] is basically constructed for highly mobility ad-hoc network and also called novel based hierarchical cluster routing protocol. This protocol uses two layers communication architecture. In layer-1, nodes forward data with each other through multi-hop path and in layer-2, nodes forward data to each other through base station.

Broadcast Based Routing Protocol [22] used broadcasting mechanism. In VANET, vehicles move with high speed and network topology change frequently so that in VANET broadcasting is very critical issue. Broadcasting used flooding technique that aims to forward information to the all nodes in the specific network.

Preferred Group Broadcast Routing Protocol (PGB) [12] is constructed to prevent the problem of broadcast storm from route request broadcasting. In PGB, each node used the sense mechanism which senses the level of signal strength which that node has shortest time out will rebroadcast the message. This protocol reduced the number of RREQ broadcasting but it is not reliable broadcasting protocol.

Distributed Vehicular Broadcast Routing Protocol (DVCAST) [14] is a broadcast based routing protocol that utilizes the information of topology by forwarding the "hello" message for transmitting the information. In DV-CAST, for checking the redundancy of packet whether it is redundant or not will use the flag variable. This protocol separates the vehicles into three types: first type is local connected (local connected uses persistence scheme like weighted p-persistent, p-persistent and slotted 1),second type is sparsely connected (In sparsely connected, when a vehicle received a broadcast message it will rebroadcast to the vehicles which moving in the same direction) and third type is totally disconnected neighborhood (this type used to store rebroadcast message until another vehicle access into transmission range and it will discard or drop the packet if time expires). In DV-CAST, the chances of network collision and broadcast storms are high because all nodes receive and broadcast the message almost at the same time.

Urban Multi-hop Broadcasting Routing Protocol (UMB) [12] is basically constructed to overcome the problem of packet collision and hidden node for the same time of message distribution in multi-hop broadcast. UMB protocol does not use any previous topology knowledge for packet forwarding and packet acknowledging. Source node tries to select farthest node in the broadcast direction. UMB protocol performs well in high vehicle traffic density and higher packet loss.

Vector Based Tracing Detection Routing Protocol (V-TRADE) [8] is based on the location and movement information of the vehicle and also it is GPS based message broadcasting protocol. In V-TRADE, each node divides their neighbors into different forwarding groups. When source node sends message to the neighboring nodes, the nodes assign only less border nodes of each group to forward the message. It utilized the better bandwidth because this used lesser number of nodes for multi-hopping. 
Edge-Aware Epidemic Routing Protocol (EAEP) [14] is a special type of reliable highly dynamic broadcast routing protocol. This protocol is used to eliminate the 'hello' message for message transfer between different clusters of vehicles and due to that it reduced the control packet overhead. To eliminate beacon message each vehicle will piggybacks its own geographical position in broadcast message and when a node receive the rebroadcast message EAEP protocol calculates the probability for making decision whether nodes will rebroadcast the message or not. For calculating this probability of making decision it will use number of transmission from front and back nodes in the given time period. In EAEP, a node does not know whether it or its neighbors have missed any messages.

Secure Ring Broadcast Routing Protocol (SRB) [14] is used to prov-ide more stable route by eliminating the number of retransmission message. In SRB, nodes are classified into three groups on the base of their receiver power. These are inner node (nodes that are closer to the sender node are called inner nodes), outer node (nodes that are far from the sender node are called outer nodes) and last one are secure ring node (nodes that are preferable distance from the sending node are called secure ring nodes).
Parameter Less Broadcasting in Static to Highly Mobile Wireless Ad-hoc Routing protocol (PBSB) [8] is the adaptive type of broadcasting protocol. In this, a node does not know about the information of location and movement of itself and its neighbor. This protocol using connects dominating sets (CDS) and neighbor elimination concepts to eliminate the redundant broadcasting. In PBSB, each vehicle maintains two lists of neighboring vehicles: $\mathrm{R}$ and NR. These help to comprising neighbors that they have already received the packet and have not received the packet.

BROADCOMM Routing Protocol [22] is a routing protocol based on hierarchical structure for highway network. In BROADCOMM, the highway protocol can be separated into virtual cells which act like vehicles. The nodes organized into two level hierarchies in the highway. In the first level all the nodes can communicate to each other and second level are cell reflectors \& can communicate with members or members of neighboring cells that are in communication range.

\section{COMPARISON OF ROUTING PROTOCOL}

The various protocols are compared based on some important parameters and requirement in the given table 1.

Table 1: Comparison of Routing Protocol

\begin{tabular}{|c|c|c|c|c|c|c|c|}
\hline Protocols & $\begin{array}{c}\text { Routing } \\
\text { Mechanis } \\
\text { m }\end{array}$ & $\begin{array}{c}\text { Digital } \\
\text { Map } \\
\text { Required }\end{array}$ & Scenario & $\begin{array}{c}\text { Position } \\
\text { Verification }\end{array}$ & Clustering & $\begin{array}{c}\text { Forwarding } \\
\text { Strategy }\end{array}$ & $\begin{array}{c}\text { Control } \\
\text { Overhead }\end{array}$ \\
\hline CGSR & Unicast & - & Urban & No & Yes & Multi-hop & - \\
\hline DSDV & Unicast & No & Urban & No & No & Multi-hop & Medium \\
\hline OLSR & Broadcast & No & Urban & No & No & Multi-hop & High \\
\hline FSR & Unicast & No & Urban & No & No & Multi-hop & High \\
\hline AODV & $\begin{array}{c}\text { Unicast } \\
\text { Multicast }\end{array}$ & No & Urban & No & No & Store \& forwarding & Low \\
\hline DSR & Unicast & No & Urban & No & No & Multi-hop & Low \\
\hline DYMO & Unicast & - & Urban & No & No & Multi-hop & - \\
\hline TORA & $\begin{array}{l}\text { Unicast } \\
\text { Multicast }\end{array}$ & No & Urban & No & No & Multi-hop & Low \\
\hline ZRP & Broadcast & No & Urban & No & - & Multi-hop & Medium \\
\hline HARP & - & No & Urban & Yes & - & Multi-hop & Medium \\
\hline GPCR & Unicast & Yes & Urban & Yes & No & Store \& Forwarding & Medium \\
\hline GPSR & Unicast & Yes & Both & Yes & No & Store \& Forwarding & Medium \\
\hline CAR & $\begin{array}{l}\text { Broadcast } \\
\text { Unicast }\end{array}$ & Yes & Both & - & No & Greedy Forwarding & High \\
\hline GSR & Unicast & Yes & Urban & Yes & No & Store \& Forwarding & Medium \\
\hline
\end{tabular}




\begin{tabular}{|c|c|c|c|c|c|c|c|}
\hline A-STAR & Unicast & Yes & Urban & Yes & No & Greedy Forwarding & - \\
\hline $\mathrm{CBF}$ & Unicast & - & Urban & Yes & No & Greedy Forwarding & Medium \\
\hline STBR & Hybrid & Yes & Highway & Yes & No & Greedy Forwarding & - \\
\hline B-MFR & Unicast & - & Urban & Yes & No & Greedy Forwarding & - \\
\hline IVG & Geo-cast & No & Highway & Yes & No & Greedy Forwarding & Low \\
\hline ROVER & Geo-cast & No & Both & - & - & Multi-hop & High \\
\hline DSTG & Multicast & No & highway & No & No & Flooding & Medium \\
\hline DG-CASTOR & Geo-cast & No & Urban & No & - & Flooding & - \\
\hline DRG & Geo-cast & No & Highway & No & - & Greedy Forwarding & - \\
\hline COIN & Unicast & Yes & - & Yes & Yes & - & - \\
\hline CBDRP & Unicast & Yes & - & - & Yes & Multi-hop & Medium \\
\hline CBR & Unicast & Yes & Urban & - & Yes & Multi-hop & Low \\
\hline CBLR & $\begin{array}{c}\text { Cluster } \\
\text { Based }\end{array}$ & Yes & Urban & - & Yes & Multi-hop & Low \\
\hline HCB & $\begin{array}{c}\text { Cluster } \\
\text { Based }\end{array}$ & Yes & Urban & - & Yes & Multi-hop & Medium \\
\hline PGB & Unicast & - & Urban & - & No & - & - \\
\hline DV-CAST & Broadcast & No & Highway & No & No & Store \& Forwarding & High \\
\hline UMB & Broadcast & - & Urban & Yes & No & - & - \\
\hline V-TRADE & Broadcast & No & Highway & No & & Flooding & High \\
\hline EAEP & Broadcast & No & Highway & No & Yes & Store \& Forwarding & High \\
\hline SRB & Broadcast & No & Highway & No & No & Store \& Forwarding & High \\
\hline PBSB & Broadcast & No & Highway & No & No & Store \& Forwarding & High \\
\hline BROADCOMM & Broadcast & - & Highway & Yes & No & - & - \\
\hline
\end{tabular}

\section{CONCLUSION \& FUTURE WORK}

VANET is self-configure network that play major role in intelligent transport system (ITS). The main aim of VANET is to provide safety in vehicular system and save lives. In VANET, the topology of the network changes very fast so that designing an efficient routing protocol is very difficult task. Routing is very important component in VANET communication.

The performance of routing protocol is depending on the movement of vehicles, driving environment and many more.

In this paper we performed a comprising survey and compare different routing protocol using some parameters. In this paper we analyzed that cluster based routing protocol and position based routing protocol is more suitable routing protocol for VANET environment.

In Topology based protocol and broadcast based protocol, the network overhead is high. So this problem provides the great opportunities in the future.

\section{REFRENCES}

[1] Fan, L. and W. Yu, Routing in vehicular ad hoc networks: A survey. Vehicular Technology Magazine, IEEE, 2007. 2(2): pp. 12-22.

[2] Kumar, R. and M. Dave, A Review of Various VANET Data Dissemination Protocols. International Journal of U-\& E-Service, Science \& Technology, 2012. 5(3). 
[3] Sharef, B.T., et al. A comparison of various vehicular ad hoc routing protocols based on communication environments. in Proceedings of the 7 th International Conference on Ubiquitous Information Management and Communication. 2013: ACM.

[4] Sharma, N. and J. Thakur, Performance analysis of AODV \&GPSR routing protocol in VANET.

[5] Khatri, M., An insight overview of issues and challenges in vehicular adhoc network. Journal of Global Research in Computer Science, 2011. 2(12): pp. 47-50.

[6] Karimi, M., Quality of Service (QoS) Provisioning in Mobile Ad-Hoc Networks (MANETs). Mobile Ad-Hoc Networks: Protocol Design, 2011.

[7] Saharan, S. and R. Kumar, QoS provisioning in vanets using mobile agent. International Journal of Computer Science and Communication, 2010. 1(1): pp. 199-202.

[8] Paul, B. and M.J. Islam, Survey over VANET Routing Protocols for Vehicle to Vehicle Communication. IOSR Journal of Computer Engineering (IOSRJCE):p p. 22780661.

[9] Allal, S. and S. Boudjit, Geocast Routing Protocols for VANETs: Survey and Geometry-Driven Scheme Proposal. Journal of Internet Services and Information Security (JISIS), 2013. 3(1/2):pp. 20-36.

[10] Bhuvaneshwari.S1, et al., A SURVEY ON VEHICULAR AD-HOC NETWORK. International Journal of Advanced Research in Electrical, Electronics and Instrumentation Engineering, 2013. Vol. 2, (Issue 10, .

[11] Altayeb, M. and I. Mahgoub, A Survey of Vehicular Ad hoc Networks Routing Protocols. International Journal of Innovation and Applied Studies, 2013. 3(3): pp. 829-846.

[12] Agarwal, U. and M. Saxena, Comparative and Behavioral Study of Various Routing Protocols in VANET. International Journal, 2013. 3(10).

[13] Sujatha, S. and P. Soundeswari, Comparative and Behavioral Study on VANET Routing Protocols: ISSN
[14] Kakarla, J., et al., A Survey on Routing Protocols and its Issues in VANET. International Journal of Computer Applications, 2011. 28.

[15] Wahid, A., H. Yoo, and D. Kim. Unicast geographic routing protocols for inter-vehicle communications: a survey. in Proceedings of the 5th ACM workshop on Performance monitoring and measurement of heterogeneous wireless and wired networks. 2010: ACM.

[16] Tee, C. and A.C.R. Lee. Survey of position based routing for inter vehicle communication system. in Distributed Framework and Applications, 2008. DFmA 2008. First International Conference on. 2008: IEEE.

[17] Nithya Darisini, P.S. and N.S. Kumari. A survey of routing protocols for VANET in urban scenarios. in Pattern Recognition, Informatics and Medical Engineering (PRIME), 2013 International Conference on: IEEE.

[18] Chennikara-Varghese, J., et al. Survey of routing protocols for inter-vehicle communications. in Mobile and Ubiquitous Systems: Networking \& Services, 2006 Third Annual International Conference on. 2006: IEEE.

[19] Paul, B., et al., VANET Routing Protocols: Pros and Cons. International Journal of Computer Applications, 2011. 20.

[20] Dhamal, P., U. Nagaraj, and D. Devotale, Study of Various Routing Protocols in VANET. 2011.

[21] Ghafoor, H. and K. Aziz. Position-based and geocast routing protocols in VANETs. in Emerging Technologies (ICET), 2011 7th International Conference on. 2011: IEEE.

[22] Spaho, E., et al. VANET Simulators: A Survey on Mobility and Routing Protocols. in Broadband and Wireless Computing, Communication and Applications (BWCCA), 2011 International Conference on. 2011: IEEE. 